Supporting information for

\title{
Influences of Longitudinal Heterogeneity on Nitrous Oxide Production from Membrane-Aerated Biofilm Reactor: A Modeling Perspective
}

Xueming Chen ${ }^{1 *}$, Linyan Yang ${ }^{2}$, Jing $\mathrm{Sun}^{3}$, Wei Wei ${ }^{4}$, Yiwen Liu ${ }^{4}$, Bing-Jie Ni*

${ }^{1}$ College of Environment and Resources, Fuzhou University, Fujian 350116, China

${ }^{2}$ School of Resources and Environmental Engineering, East China University of Science and Technology, Shanghai 200237, China

${ }^{3}$ State Key Laboratory of Pollution Control and Resources Reuse, College of Environmental Science and Engineering, Tongji University, Shanghai 200092, China

${ }^{4}$ Centre for Technology in Water and Wastewater, School of Civil and Environmental Engineering, University of Technology Sydney, Sydney, NSW 2007, Australia

\section{*Corresponding authors:}

Dr. Xueming Chen, E-mail: xuem.chen@ hotmail.com

Dr. Bing-Jie Ni, E-mail: bingjieni@gmail.com

Number of pages: 13

Number of figures: 3

Number of tables: 5 


\section{Axial $\mathrm{N}_{2} \mathrm{O}$ Production Profiles}

As indicated by the comparable trends in Fig. 5, the number of compartments wouldn't affect the longitudinal trends of $\mathrm{N}_{2} \mathrm{O}$ production. Therefore, only the axial $\mathrm{DO}$ and $\mathrm{N}_{2} \mathrm{O}$ production profiles for Configurations 2-1, 3-1 and 4-3 were presented and compared to those for the traditional modeling configuration of the MABR (i.e., Configuration 1-0). This was to reveal the biofilm thickness-wise difference between modeling approaches, and the results are demonstrated in Fig. S2.

As shown in Fig. S2A, net $\mathrm{N}_{2} \mathrm{O}$ turnover (i.e., production or consumption) took place in both the aerobic and anoxic zones at the base of the biofilm for Configuration 1-0. Comparatively, Configuration 2-1 not only significantly dwarfed the magnitude of the $\mathrm{N}_{2} \mathrm{O}$ turnover rate but also limited the net $\mathrm{N}_{2} \mathrm{O}$ turnover to the aerobic zone (see Fig. S2B). This also demonstrated the important role played by the longitudinal gradient in oxygen in affecting the simulated $\mathrm{N}_{2} \mathrm{O}$ production of the MABR. As shown in Fig. S2C, the axial profiles of both $\mathrm{N}_{2} \mathrm{O}$ turnover rate and DO for Configuration 3-1 were similar to those for Configuration 1-0, and the only slight discrepancy was found between the $\mathrm{N}_{2} \mathrm{O}$ turnover profiles of different compartments at the biofilm base. As shown in Fig. S2D, the introduction of the longitudinal gradient in biofilm thickness greatly complicated the simulated axial $\mathrm{N}_{2} \mathrm{O}$ turnover profiles. For the first two compartments with a higher biofilm thickness, the axial profiles of $\mathrm{N}_{2} \mathrm{O}$ turnover rates resembled those in Fig. S2A and C. By contrast, due to the penetration of oxygen, there was only net $\mathrm{N}_{2} \mathrm{O}$ production across the biofilm in the last compartment with the thinnest biofilm (see Fig. S2D). 
Overall, these axial profiles shown in Fig. $\mathbf{S 2}$ were to a large extent consistent with the experimental observations of an MABR treating synthetic industrial wastewater reported by Kinh et al. ${ }^{1}$ in the following:

1. DO was rapidly consumed at the biofilm base close to the membrane surface.

2. $\mathrm{N}_{2} \mathrm{O}$ production mainly occurred at the bottom of the biofilm.

3. Hotspots for $\mathrm{N}_{2} \mathrm{O}$ consumption emerged adjacent to the region for $\mathrm{N}_{2} \mathrm{O}$ production. 


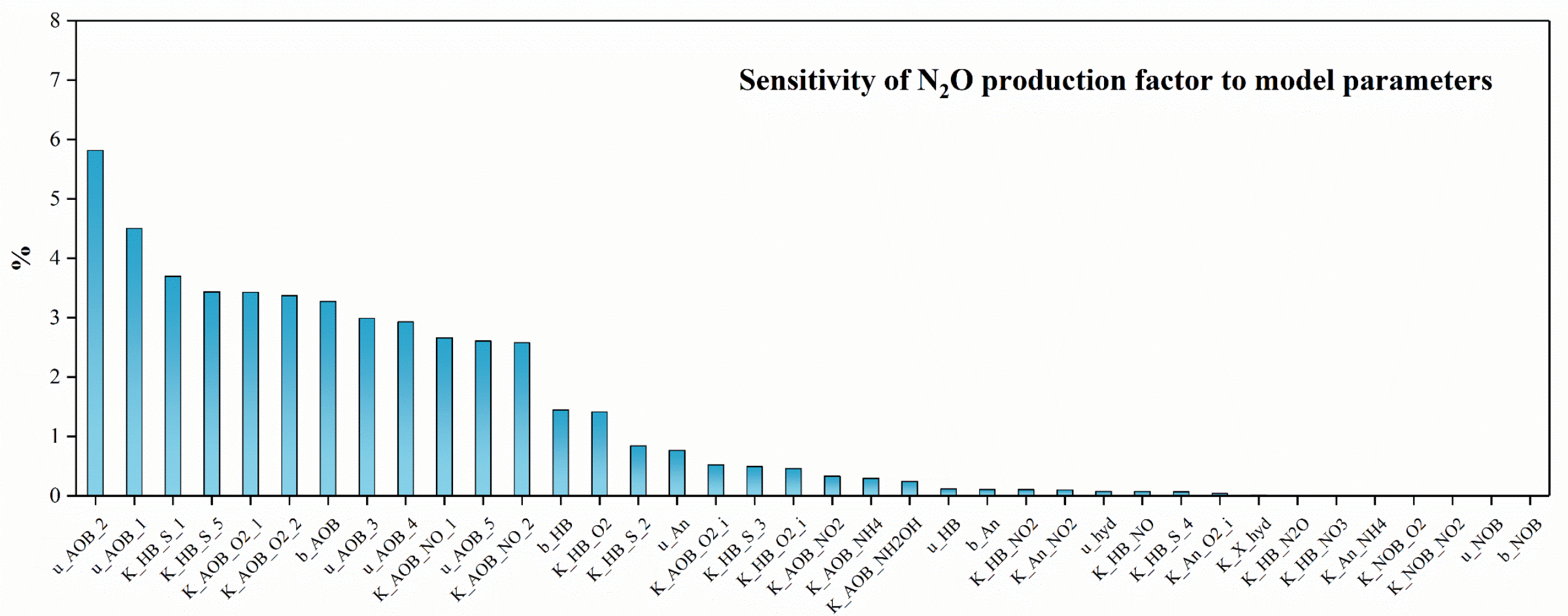

Figure S1. Sensitivity of $\mathrm{N}_{2} \mathrm{O}$ production factor to model parameters under the studied operational conditions of the membrane-aerated biofilm reactor (MABR). 

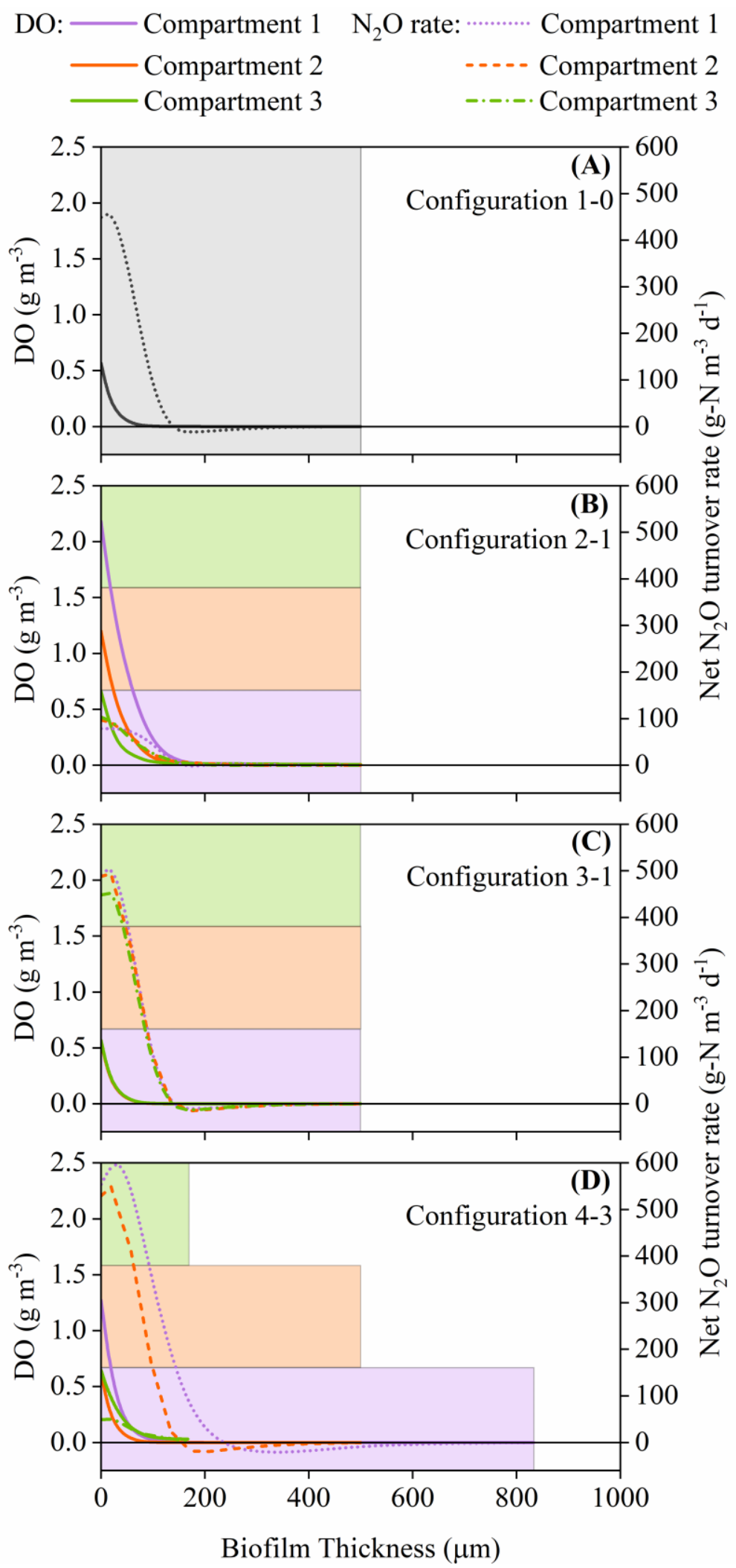

Figure S2. Comparison of axial (thickness-wise) profiles of dissolved oxygen (DO) and net $\mathrm{N}_{2} \mathrm{O}$ turnover rate for the simulated membrane-aerated biofilm reactor Configuration (A) 1-0, (B) 2-1, (C) 3-1, and (D) 4-3. 


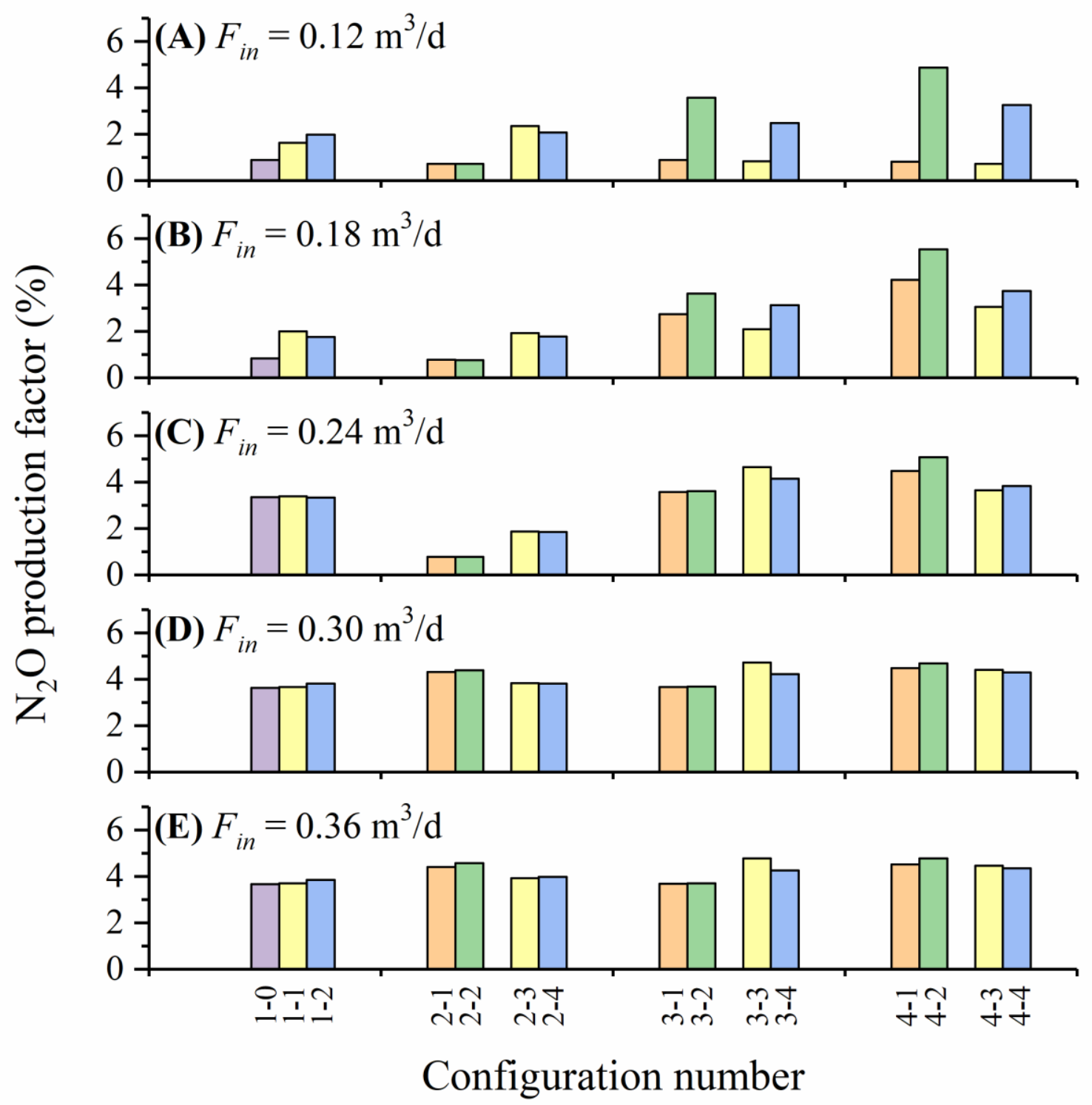

Figure S3. $\mathrm{N}_{2} \mathrm{O}$ production factors of all the simulated membrane-aerated biofilm reactor (MABR) configurations at influent flow rate $\left(F_{\text {in }}\right)$ of $(\mathbf{A}) 0.12 \mathrm{~m}^{3} / \mathrm{d},(\mathbf{B}) 0.18 \mathrm{~m}^{3} / \mathrm{d},(\mathbf{C}) 0.24$ $\mathrm{m}^{3} / \mathrm{d},(\mathbf{D}) 0.30 \mathrm{~m}^{3} / \mathrm{d}$, and (E) $0.36 \mathrm{~m}^{3} / \mathrm{d}$. 
Table S1. Component definitions of the model

\begin{tabular}{|c|c|c|}
\hline Variable & Description & Unit \\
\hline \multicolumn{3}{|c|}{ Solid-phase components } \\
\hline$X_{A O B}$ & Ammonium-oxidizing bacteria (AOB) concentration & $\mathrm{g}-\mathrm{COD} / \mathrm{m}^{3}$ \\
\hline$X_{N O B}$ & Nitrite-oxidizing bacteria (NOB) concentration & $\mathrm{g}-\mathrm{COD} / \mathrm{m}^{3}$ \\
\hline$X_{H B}$ & Heterotrophic bacteria $(\mathrm{HB})$ concentration & $\mathrm{g}-\mathrm{COD} / \mathrm{m}^{3}$ \\
\hline$X_{A n}$ & Anaerobic ammonium oxidizing (Anammox) bacteria concentration & $\mathrm{g}-\mathrm{COD} / \mathrm{m}^{3}$ \\
\hline$X_{S}$ & Slowly biodegradable organics concentration & $\mathrm{g}-\mathrm{COD} / \mathrm{m}^{3}$ \\
\hline$X_{I}$ & Inert, non-biodegradable organics concentration & $\mathrm{g}-\mathrm{COD} / \mathrm{m}^{3}$ \\
\hline \multicolumn{3}{|c|}{ Liquid-phase components } \\
\hline$S_{S}$ & Readily biodegradable organics concentration & $\mathrm{g}-\mathrm{COD} / \mathrm{m}^{3}$ \\
\hline$S_{O 2}$ & Dissolved oxygen (DO) concentration & $\mathrm{g}-\mathrm{O}_{2} / \mathrm{m}^{3}$ \\
\hline$S_{N H 4}$ & Ammonium $\left(\mathrm{NH}_{4}^{+}\right)$concentration & $\mathrm{g}-\mathrm{N} / \mathrm{m}^{3}$ \\
\hline$S_{\mathrm{NH} 2 \mathrm{OH}}$ & Hydroxylamine $\left(\mathrm{NH}_{2} \mathrm{OH}\right)$ concentration & $\mathrm{g}-\mathrm{N} / \mathrm{m}^{3}$ \\
\hline$S_{\mathrm{NO} 2}$ & Nitrite $\left(\mathrm{NO}_{2}^{-}\right)$concentration & $\mathrm{g}-\mathrm{N} / \mathrm{m}^{3}$ \\
\hline$S_{\mathrm{NO} 3}$ & Nitrate $\left(\mathrm{NO}_{3}{ }^{-}\right)$concentration & $\mathrm{g}-\mathrm{N} / \mathrm{m}^{3}$ \\
\hline$S_{N 2 O}$ & Nitrous oxide $\left(\mathrm{N}_{2} \mathrm{O}\right)$ concentration & $\mathrm{g}-\mathrm{N} / \mathrm{m}^{3}$ \\
\hline$S_{N O}$ & Nitric oxide (NO) concentration & $\mathrm{g}-\mathrm{N} / \mathrm{m}^{3}$ \\
\hline$S_{N 2}$ & Dinitrogen $\left(\mathrm{N}_{2}\right)$ concentration & $\mathrm{g}-\mathrm{N} / \mathrm{m}^{3}$ \\
\hline
\end{tabular}


Table S2. Stoichiometric matrix of the model

\begin{tabular}{|c|c|c|c|c|c|c|c|c|c|c|c|c|c|c|c|}
\hline $\begin{array}{l}\text { Components } \rightarrow \\
\text { Reactions } \downarrow\end{array}$ & Ss & $\mathrm{S}_{\mathrm{O} 2}$ & $\mathrm{~S}_{\mathrm{NO} O 3}$ & $\mathrm{~S}_{\mathrm{NO} 2}$ & SNo & $\mathrm{S}_{\mathrm{N} 20}$ & $\mathrm{~S}_{\mathrm{N} 2}$ & $\mathrm{~S}_{\mathrm{NH} 4}$ & $\mathrm{~S}_{\mathrm{NH} 2 \mathrm{OH}}$ & $\mathbf{X}_{\mathrm{AOB}}$ & $\mathbf{X}_{\mathrm{NOB}}$ & $\mathbf{X}_{\mathrm{HB}}$ & $\mathbf{X}_{\mathrm{An}}$ & $\mathbf{X s}_{\mathrm{s}}$ & $\mathbf{X}_{\mathbf{I}}$ \\
\hline \multicolumn{16}{|l|}{ Heterotrophic bacteria $(\mathrm{HB})$} \\
\hline 1. Hydrolysis & 1 & & & & & & & & & & & & & -1 & \\
\hline 2. Aerobic growth & $-\frac{1}{\mathrm{Y}_{\mathrm{HB}}}$ & $-\frac{1-\mathrm{Y}_{\mathrm{HB}}}{\mathrm{Y}_{\mathrm{HB}}}$ & & & & & & $-i_{\mathrm{xbn}}$ & & & & 1 & & & \\
\hline 3. Anoxic $\mathrm{NO}_{3}^{-}$reduction to $\mathrm{NO}_{2}^{-}$ & $-\frac{1}{Y_{H B}}$ & & $-\frac{1-\mathrm{Y}_{\mathrm{HB}}}{1.143 \mathrm{Y}_{\mathrm{HB}}}$ & $\frac{1-\mathrm{Y}_{\mathrm{HB}}}{1.143 \mathrm{Y}_{\mathrm{HB}}}$ & & & & $-i_{x b n}$ & & & & 1 & & & \\
\hline 4. Anoxic $\mathrm{NO}_{2}^{-}$reduction to $\mathrm{NO}$ & $-\frac{1}{Y_{H B}}$ & & & $-\frac{1-\mathrm{Y}_{\mathrm{HB}}}{0.571 \mathrm{Y}_{\mathrm{HB}}}$ & $\frac{1-\mathrm{Y}_{\mathrm{HB}}}{0.571 \mathrm{Y}_{\mathrm{HB}}}$ & & & $-i_{\mathrm{xbn}}$ & & & & 1 & & & \\
\hline 5. Anoxic $\mathrm{NO}$ reduction to $\mathrm{N}_{2} \mathrm{O}$ & $-\frac{1}{\mathrm{Y}_{\mathrm{HB}}}$ & & & & $-\frac{1-\mathrm{Y}_{\mathrm{HB}}}{0.571 \mathrm{Y}_{\mathrm{HB}}}$ & $\frac{1-\mathrm{Y}_{\mathrm{HB}}}{0.571 \mathrm{Y}_{\mathrm{HB}}}$ & & $-i_{x b n}$ & & & & 1 & & & \\
\hline 6. Anoxic $\mathrm{N}_{2} \mathrm{O}$ reduction to $\mathrm{N}_{2}$ & $-\frac{1}{Y_{H B}}$ & & & & & $-\frac{1-\mathrm{Y}_{\mathrm{HB}}}{0.571 \mathrm{Y}_{\mathrm{HB}}}$ & $\frac{1-\mathrm{Y}_{\mathrm{HB}}}{0.571 \mathrm{Y}_{\mathrm{HB}}}$ & $-i_{x b n}$ & & & & 1 & & & \\
\hline 7. Decay & & & & & & & & $i_{x b n}-f_{I} \cdot i_{x i n}$ & & & & -1 & & $1-f_{I}$ & $\mathrm{f}_{\mathrm{I}}$ \\
\hline \multicolumn{16}{|c|}{ Ammonium-oxidizing bacteria $(A O B)$} \\
\hline 8. $\mathrm{NH}_{4}{ }^{+}$oxidation to $\mathrm{NH}_{2} \mathrm{OH}$ & & -1.143 & & & & & & -1 & 1 & & & & & & \\
\hline 10. $\mathrm{NO}$ oxidation to $\mathrm{NO}_{2}^{-}$ & & -0.571 & & 1 & -1 & & & & & & & & & & \\
\hline 11. $\mathrm{NO}$ reduction to $\mathrm{N}_{2} \mathrm{O}$ & & & & 1 & -4 & 4 & & & -1 & & & & & & \\
\hline 12. $\mathrm{NO}_{2}^{-}$reduction to $\mathrm{N}_{2} \mathrm{O}$ & & & & -1 & & 2 & & & -1 & & & & & & \\
\hline 13. Decay & & & & & & & & $\mathrm{i}_{\mathrm{xbn}}-\mathrm{f}_{\mathrm{I}} \cdot \mathrm{i}_{\mathrm{xin}}$ & & -1 & & & & $1-f_{I}$ & $\mathrm{f}_{\mathrm{I}}$ \\
\hline \multicolumn{16}{|c|}{ Nitrite-oxidizing bacteria (NOB) } \\
\hline 14. $\mathrm{NO}_{2}^{-}$oxidation to $\mathrm{NO}_{3}^{-}$ & & $-\frac{1.143-\mathrm{Y}_{\mathrm{NOB}}}{\mathrm{Y}_{\mathrm{NOB}}}$ & $\frac{1}{\mathrm{Y}_{\mathrm{NOB}}}$ & $-\frac{1}{\mathrm{Y}_{\mathrm{NOB}}}$ & & & & $-i_{x b n}$ & & & 1 & & & & \\
\hline 15. Decay & & & & & & & & $i_{x b n}-f_{I} \cdot i_{x i n}$ & & & -1 & & & $1-f_{I}$ & $\mathrm{f}_{\mathrm{I}}$ \\
\hline \multicolumn{16}{|c|}{ Anaerobic ammonium oxidizing (Anammox) bacteria } \\
\hline 16. Anoxic growth & & & $\frac{1}{1.14}$ & $-\frac{1}{\mathrm{Y}_{\mathrm{An}}}-\frac{1}{1.14}$ & & & $\frac{2}{Y_{\mathrm{An}}}$ & $-\mathrm{i}_{\mathrm{xbn}}-\frac{1}{\mathrm{Y}_{\mathrm{An}}}$ & & & & & 1 & & \\
\hline 17. Decay & & & & & & & & $\mathrm{i}_{\mathrm{xbn}}-\mathrm{f}_{\mathrm{I}} \cdot \mathrm{i}_{\mathrm{xin}}$ & & & & & -1 & $1-f_{I}$ & $\mathrm{f}_{\mathrm{I}}$ \\
\hline
\end{tabular}


Table S3. Process kinetic rate equations of the model

\begin{tabular}{|c|c|}
\hline Process & Kinetic rate equation \\
\hline \multicolumn{2}{|l|}{ Heterotrophic bacteria $(\mathrm{HB})$} \\
\hline 1. Hydrolysis & $\mu_{h y d} \frac{X_{S}}{X_{S}+X_{H B} K_{X, h y d}} X_{H B}$ \\
\hline 2. Aerobic growth & $\mu_{H B} \frac{S_{O 2}}{K_{O 2}^{H B}+S_{O 2}} \frac{S_{S}}{K_{S, 1}^{H B}+S_{S}} X_{H B}$ \\
\hline 3. Anoxic $\mathrm{NO}_{3}{ }^{-}$reduction to $\mathrm{NO}_{2}{ }^{-}$ & $\mu_{H B} \eta_{H B, 1} \frac{K_{O 2, i}^{H B}}{K_{O 2, i}^{H B}+S_{O 2}} \frac{S_{S}}{K_{S, 2}^{H B}+S_{S}} \frac{S_{N O 3}}{K_{N O 3}^{H B}+S_{N O 3}} X_{H B}$ \\
\hline 4. Anoxic $\mathrm{NO}_{2}^{-}$reduction to $\mathrm{NO}$ & $\mu_{H B} \eta_{H B, 2} \frac{K_{O 2, i}^{H B}}{K_{O 2, i}^{H B}+S_{O 2}} \frac{S_{S}}{K_{S, 3}^{H B}+S_{S}} \frac{S_{N O 2}}{K_{N O 2}^{H B}+S_{N O 2}} X_{H B}$ \\
\hline 5. Anoxic $\mathrm{NO}$ reduction to $\mathrm{N}_{2} \mathrm{O}$ & $\mu_{H B} \eta_{H B, 3} \frac{K_{O 2, i}^{H B}}{K_{O 2, i}^{H B}+S_{O 2}} \frac{S_{S}}{K_{S, 4}^{H B}+S_{S}} \frac{S_{N O}}{K_{N O}^{H B}+S_{N O}} X_{H B}$ \\
\hline 6. Anoxic $\mathrm{N}_{2} \mathrm{O}$ reduction to $\mathrm{N}_{2}$ & $\mu_{H B} \eta_{H B, 4} \frac{K_{O 2, i}^{H B}}{K_{O 2, i}^{H B}+S_{O 2}} \frac{S_{S}}{K_{S, 5}^{H B}+S_{S}} \frac{S_{N 2 O}}{K_{N 2 O}^{H B}+S_{N 2 O}} X_{H B}$ \\
\hline 7. Decay & $b_{H B} X_{H B}$ \\
\hline \multicolumn{2}{|c|}{ Ammonium-oxidizing bacteria $(\mathrm{AOB})$} \\
\hline 8. $\mathrm{NH}_{4}{ }^{+}$oxidation to $\mathrm{NH}_{2} \mathrm{OH}$ & $\mu_{A O B, 1} \frac{S_{O 2}}{K_{O 2,1}^{A O B}+S_{O 2}} \frac{S_{N H 4}}{K_{N H 4}^{A O B}+S_{N H 4}} X_{A O B}$ \\
\hline 9. $\mathrm{NH}_{2} \mathrm{OH}$ oxidation to $\mathrm{NO}$ & $\mu_{A O B, 2} \frac{S_{O 2}}{K_{O 2,2}^{A O B}+S_{O 2}} \frac{S_{N H 2 O H}}{K_{N H 2 O H}^{A O B}+S_{N H 2 O H}} X_{A O B}$ \\
\hline 10. $\mathrm{NO}$ oxidation to $\mathrm{NO}_{2}^{-}$ & $\mu_{A O B, 3} \frac{S_{O 2}}{K_{O 2,2}^{A O B}+S_{O 2}} \frac{S_{N O}}{K_{N O, 1}^{A O B}+S_{N O}} X_{A O B}$ \\
\hline 11. $\mathrm{NO}$ reduction to $\mathrm{N}_{2} \mathrm{O}$ & $\mu_{A O B, 4} \frac{S_{N H 2 O H}}{K_{N H 2 O H}^{A O B}+S_{N H 2 O H}} \frac{S_{N O}}{K_{N O, 2}^{A O B}+S_{N O}} X_{A O B}$ \\
\hline 12. $\mathrm{NO}_{2}^{-}$reduction to $\mathrm{N}_{2} \mathrm{O}$ & $\mu_{A O B, 5} \frac{K_{O 2, i}^{A O B}}{K_{O 2, i}^{A O B}+S_{O 2}} \frac{S_{N H 2 O H}}{K_{N H 2 O H}^{A O B}+S_{N H 2 O H}} \frac{S_{N O 2}}{K_{N O 2}^{A O B}+S_{N O 2}} X_{A O B}$ \\
\hline 13. Decay & $b_{A O B} X_{A O B}$ \\
\hline \multicolumn{2}{|l|}{ Nitrite-oxidizing bacteria (NOB) } \\
\hline 14. $\mathrm{NO}_{2}^{-}$oxidation to $\mathrm{NO}_{3}^{-}$ & $\mu_{N O B} \frac{S_{N O 2}}{K_{N O 2}^{N O B}+S_{N O 2}} \frac{S_{O 2}}{K_{O 2}^{N O B}+S_{O 2}} X_{N O B}$ \\
\hline 15. Decay & $b_{N O B} X_{N O B}$ \\
\hline \multicolumn{2}{|c|}{ Anaerobic ammonium oxidizing (Anammox) bacteria } \\
\hline 16. Anoxic growth & $\mu_{A n} \frac{K_{O 2, i}^{A n}}{K_{O 2, i}^{A n}+S_{O 2}} \frac{S_{N H 4}}{K_{N H 4}^{A n}+S_{N H 4}} \frac{S_{N O 2}}{K_{N O 2}^{A n}+S_{N O 2}} X_{A n}$ \\
\hline 17. Decay & $b_{A n} X_{A n}$ \\
\hline
\end{tabular}


Table S4. Stoichiometric and kinetic parameters of the model

\begin{tabular}{|c|c|c|c|c|}
\hline Parameter & Definition & Value & Unit & Source \\
\hline \multicolumn{5}{|c|}{ Stoichiometric parameters } \\
\hline$Y_{H B}$ & Yield coefficient for $\mathrm{HB}$ & 0.60 & g-COD/g-COD & 2 \\
\hline$Y_{A O B}$ & Yield coefficient for AOB & 0.18 & $\mathrm{~g}-\mathrm{COD} / \mathrm{g}-\mathrm{N}$ & 2 \\
\hline$Y_{N O B}$ & Yield coefficient for NOB & 0.06 & $\mathrm{~g}-\mathrm{COD} / \mathrm{g}-\mathrm{N}$ & 2 \\
\hline$Y_{A n}$ & Yield coefficient for Anammox & 0.16 & $\mathrm{~g}-\mathrm{COD} / \mathrm{g}-\mathrm{N}$ & 3 \\
\hline$f_{I}$ & Fraction of $X_{I}$ in biomass decay & 0.08 & $\mathrm{~g}-\mathrm{COD} / \mathrm{g}-\mathrm{COD}$ & 2 \\
\hline$i_{x b n}$ & Nitrogen content of active biomass & 0.086 & g-N/g-COD & 2 \\
\hline$i_{x i n}$ & Nitrogen content of inert biomass & 0.06 & $\mathrm{~g}-\mathrm{N} / \mathrm{g}-\mathrm{COD}$ & 2 \\
\hline \multicolumn{5}{|c|}{ Heterotrophic bacteria $(\mathrm{HB})$} \\
\hline$\mu_{H B}$ & Maximum specific growth rate & 6.25 & $1 / \mathrm{d}$ & 2 \\
\hline$\eta_{H B, 1}$ & $\begin{array}{l}\text { Anoxic growth factor } 1 \text { (for } \mathrm{NO}_{3}^{-} \text {reduction to } \\
\mathrm{NO}_{2}^{-} \text {) }\end{array}$ & 0.28 & - & 2 \\
\hline$\eta_{H B, 2}$ & $\begin{array}{l}\text { Anoxic growth factor } 2 \text { (for } \mathrm{NO}_{2}^{-} \text {reduction to } \\
\mathrm{NO} \text { ) }\end{array}$ & 0.16 & - & 2 \\
\hline$\eta_{H B, 3}$ & $\begin{array}{l}\text { Anoxic growth factor } 3 \text { (for } \mathrm{NO} \text { reduction to } \\
\mathrm{N}_{2} \mathrm{O} \text { ) }\end{array}$ & 0.35 & - & 2 \\
\hline$\eta_{H B, 4}$ & Anoxic growth factor 4 (for $\mathrm{N}_{2} \mathrm{O}$ reduction to $\mathrm{N}_{2}$ ) & 0.35 & - & 2 \\
\hline$\mu_{\text {hyd }}$ & Maximum specific hydrolysis rate & 2.208 & $1 / \mathrm{d}$ & 2 \\
\hline$K_{X, h y d}$ & $\mathrm{X}_{\mathrm{S}}$ affinity constant & 0.15 & g-COD/g-COD & 2 \\
\hline$K_{S, 1}^{H B}$ & $\mathrm{~S}_{\mathrm{S}}$ affinity constant 1 (for aerobic growth) & 20 & $\mathrm{~g}-\mathrm{COD} / \mathrm{m}^{3}$ & 2 \\
\hline$K_{S, 2}^{H B}$ & $\mathrm{~S}_{\mathrm{S}}$ affinity constant 2 (for $\mathrm{NO}_{3}^{-}$reduction to $\mathrm{NO}_{2}^{-}$) & 20 & $\mathrm{~g}-\mathrm{COD} / \mathrm{m}^{3}$ & 2 \\
\hline$K_{S, 3}^{H B}$ & $\mathrm{~S}_{\mathrm{S}}$ affinity constant 3 (for $\mathrm{NO}_{2}^{-}$reduction to $\mathrm{NO}$ ) & 20 & $\mathrm{~g}-\mathrm{COD} / \mathrm{m}^{3}$ & 2 \\
\hline$K_{S, 4}^{H B}$ & $\mathrm{~S}_{\mathrm{S}}$ affinity constant 4 (for $\mathrm{NO}$ reduction to $\mathrm{N}_{2} \mathrm{O}$ ) & 20 & $\mathrm{~g}-\mathrm{COD} / \mathrm{m}^{3}$ & 2 \\
\hline$K_{S, 5}^{H B}$ & $\mathrm{~S}_{\mathrm{S}}$ affinity constant 5 (for $\mathrm{N}_{2} \mathrm{O}$ reduction to $\mathrm{N}_{2}$ ) & 40 & $\mathrm{~g}-\mathrm{COD} / \mathrm{m}^{3}$ & 2 \\
\hline$K_{N O 3}^{H B}$ & $\mathrm{NO}_{3}{ }^{-}$affinity constant & 0.2 & $\mathrm{~g}-\mathrm{N} / \mathrm{m}^{3}$ & 2 \\
\hline$K_{N O 2}^{H B}$ & $\mathrm{NO}_{2}^{-}$affinity constant & 0.2 & $\mathrm{~g}-\mathrm{N} / \mathrm{m}^{3}$ & 2 \\
\hline$K_{N O}^{H B}$ & NO affinity constant & 0.05 & $\mathrm{~g}-\mathrm{N} / \mathrm{m}^{3}$ & 2 \\
\hline$K_{N 2 O}^{H B}$ & $\mathrm{~N}_{2} \mathrm{O}$ affinity constant & 0.05 & $\mathrm{~g}-\mathrm{N} / \mathrm{m}^{3}$ & 2 \\
\hline$K_{O 2}^{H B}$ & $\mathrm{O}_{2}$ affinity constant & 0.1 & $\mathrm{~g}-\mathrm{O}_{2} / \mathrm{m}^{3}$ & 2 \\
\hline$K_{O 2, i}^{H B}$ & $\mathrm{O}_{2}$ inhibition constant & 0.1 & $\mathrm{~g}-\mathrm{O}_{2} / \mathrm{m}^{3}$ & 2 \\
\hline$b_{H B}$ & Decay rate & 0.408 & $1 / \mathrm{d}$ & 2 \\
\hline \multicolumn{5}{|c|}{ Ammonium-oxidizing bacteria $(A O B)$} \\
\hline$\mu_{A O B, 1}$ & $\begin{array}{l}\text { Maximum reaction rate for } \mathrm{NH}_{4}^{+} \text {oxidation to } \\
\mathrm{NH}_{2} \mathrm{OH}\end{array}$ & 4.92 & $\mathrm{~g}-\mathrm{N} /(\mathrm{g}-\mathrm{COD} * \mathrm{~d})$ & 4 \\
\hline$\mu_{A O B, 2}$ & Maximum specific growth rate & 2.04 & $1 / \mathrm{d}$ & 4 \\
\hline$\mu_{A O B, 3}$ & Maximum reaction rate for $\mathrm{NO}$ oxidation to $\mathrm{NO}_{2}^{-}$ & 13.61 & $\mathrm{~g}-\mathrm{N} /(\mathrm{g}-\mathrm{COD} * \mathrm{~d})$ & 4 \\
\hline$\mu_{A O B, 4}$ & Maximum reaction rate for $\mathrm{NO}$ reduction to $\mathrm{N}_{2} \mathrm{O}$ & 0.58 & $\mathrm{~g}-\mathrm{N} /(\mathrm{g}-\mathrm{COD} * \mathrm{~d})$ & 4 \\
\hline
\end{tabular}




\begin{tabular}{|c|c|c|c|c|}
\hline$\mu_{A O B, 5}$ & Maximum reaction rate for $\mathrm{NO}_{2}^{-}$reduction to $\mathrm{N}_{2} \mathrm{O}$ & 0.16 & $\mathrm{~g}-\mathrm{N} /(\mathrm{g}-\mathrm{COD} * \mathrm{~d})$ & 5 \\
\hline$K_{N H 4}^{A O B}$ & $\mathrm{NH}_{4}{ }^{+}$affinity constant & 2.4 & $\mathrm{~g}-\mathrm{N} / \mathrm{m}^{3}$ & 4 \\
\hline$K_{\mathrm{NH} 2 \mathrm{OH}}^{\mathrm{AOB}}$ & $\mathrm{NH}_{2} \mathrm{OH}$ affinity constant & 2.4 & $\mathrm{~g}-\mathrm{N} / \mathrm{m}^{3}$ & 4 \\
\hline$K_{N O, 1}^{A O B}$ & NO affinity constant 1 & 0.0084 & $\mathrm{~g}-\mathrm{N} / \mathrm{m}^{3}$ & 4 \\
\hline$K_{N O, 2}^{A O B}$ & NO affinity constant 2 & 0.0084 & $\mathrm{~g}-\mathrm{N} / \mathrm{m}^{3}$ & 4 \\
\hline$K_{N O 2}^{A O B}$ & $\mathrm{NO}_{2}^{-}$affinity constant & 0.14 & $\mathrm{~g}-\mathrm{N} / \mathrm{m}^{3}$ & 5 \\
\hline$K_{O 2,1}^{A O B}$ & $\mathrm{O}_{2}$ affinity constant 1 & 0.4 & $\mathrm{~g}-\mathrm{O}_{2} / \mathrm{m}^{3}$ & 4 \\
\hline$K_{O 2,2}^{A O B}$ & $\mathrm{O}_{2}$ affinity constant 2 & 0.073 & $\mathrm{~g}-\mathrm{O}_{2} / \mathrm{m}^{3}$ & 4 \\
\hline$K_{O 2, i}^{A O B}$ & $\mathrm{O}_{2}$ inhibition constant & 0.112 & $\mathrm{~g}-\mathrm{O}_{2} / \mathrm{m}^{3}$ & 5 \\
\hline$b_{A O B}$ & Decay rate & 0.096 & $1 / \mathrm{d}$ & 2 \\
\hline \multicolumn{5}{|c|}{ Nitrite-oxidizing bacteria (NOB) } \\
\hline$\mu_{N O B}$ & Maximum specific growth rate & 0.78 & $1 / \mathrm{d}$ & 2 \\
\hline$K_{O 2}^{N O B}$ & $\mathrm{O}_{2}$ affinity constant & 2.2 & $\mathrm{~g}-\mathrm{O}_{2} / \mathrm{m}^{3}$ & 6 \\
\hline $\mathrm{K}_{\mathrm{NO} 2}^{\mathrm{NOB}}$ & $\mathrm{NO}_{2}^{-}$affinity constant & 5.5 & $\mathrm{~g}-\mathrm{N} / \mathrm{m}^{3}$ & 6 \\
\hline$b_{N O B}$ & Decay rate & 0.096 & $1 / \mathrm{d}$ & 2 \\
\hline \multicolumn{5}{|c|}{ Anaerobic ammonium oxidizing (Anammox) bacteria } \\
\hline$\mu_{A n}$ & Maximum specific growth rate & 0.08 & $1 / \mathrm{d}$ & 7 \\
\hline$K_{N H 4}^{A n}$ & $\mathrm{NH}_{4}{ }^{+}$affinity constant & 0.07 & $\mathrm{~g}-\mathrm{N} / \mathrm{m}^{3}$ & 3 \\
\hline$K_{N O 2}^{A n}$ & $\mathrm{NO}_{2}^{-}$affinity constant & 0.05 & $\mathrm{~g}-\mathrm{N} / \mathrm{m}^{3}$ & 8 \\
\hline$K_{O 2, i}^{A n}$ & $\mathrm{O}_{2}$ inhibition constant & 0.01 & $\mathrm{~g}-\mathrm{O}_{2} / \mathrm{m}^{3}$ & 3 \\
\hline$b_{A n}$ & Decay rate & 0.003 & $1 / \mathrm{d}$ & 8 \\
\hline
\end{tabular}


Table S5. Other parameters used in the model simulations

\begin{tabular}{|c|c|c|c|c|}
\hline Parameter & Definition & Value & Unit & Source \\
\hline \multicolumn{5}{|c|}{ Generic parameters for model simulations } \\
\hline$D_{\mathrm{NO} 3}$ & $\mathrm{NO}_{3}^{-}$diffusion coefficient in water & $1.64 \times 10^{-4}$ & $\mathrm{~m}^{2} / \mathrm{d}$ & 9 \\
\hline$D_{\mathrm{NO} 2}$ & $\mathrm{NO}_{2}^{-}$diffusion coefficient in water & $1.65 \times 10^{-4}$ & $\mathrm{~m}^{2} / \mathrm{d}$ & 9 \\
\hline$D_{N 2 O}$ & $\mathrm{~N}_{2} \mathrm{O}$ diffusion coefficient in water & $2.22 \times 10^{-4}$ & $\mathrm{~m}^{2} / \mathrm{d}$ & 9 \\
\hline$D_{N 2}$ & $\mathrm{~N}_{2}$ diffusion coefficient in water & $1.73 \times 10^{-4}$ & $\mathrm{~m}^{2} / \mathrm{d}$ & 9 \\
\hline$D_{O 2}$ & $\mathrm{O}_{2}$ diffusion coefficient in water & $2.09 \times 10^{-4}$ & $\mathrm{~m}^{2} / \mathrm{d}$ & 9 \\
\hline$D_{N H 4}$ & $\mathrm{NH}_{4}{ }^{+}$diffusion coefficient in water & $1.69 \times 10^{-4}$ & $\mathrm{~m}^{2} / \mathrm{d}$ & 9 \\
\hline$D_{\mathrm{NH} 2 \mathrm{OH}}$ & $\mathrm{NH}_{2} \mathrm{OH}$ diffusion coefficient in water & $2.09 \times 10^{-4}$ & $\mathrm{~m}^{2} / \mathrm{d}$ & 10 \\
\hline$D_{N O}$ & NO diffusion coefficient in water & $2.22 \times 10^{-4}$ & $\mathrm{~m}^{2} / \mathrm{d}$ & 11 \\
\hline$D_{S}$ & S $_{\mathrm{S}}$ diffusion coefficient in water & $2.09 \times 10^{-4}$ & $\mathrm{~m}^{2} / \mathrm{d}$ & 9 \\
\hline$f$ & $\begin{array}{l}\text { Reduction factor of diffusion } \\
\text { coefficients in biofilm }\end{array}$ & 0.5 & - & 12 \\
\hline$\beta_{X}$ & Biomass density & 50000 & $\mathrm{~g}-\mathrm{COD} / \mathrm{m}^{3}$ & chosen \\
\hline$\theta$ & Porosity & 0.75 & - & 7 \\
\hline \multicolumn{5}{|c|}{ Parameters specific for model simulations } \\
\hline$V_{M A B R}$ & $\begin{array}{l}\text { Total volume of membrane-aerated } \\
\text { biofilm reactor (MABR) }\end{array}$ & 1 & $\mathrm{~m}^{3}$ & chosen \\
\hline$V_{\text {biofilm }}$ & Volume of biofilm reactor & 0.96 & $\mathrm{~m}^{3}$ & chosen \\
\hline$V_{\text {membrane }}$ & Volume of membrane lumen & 0.04 & $\mathrm{~m}^{3}$ & calculated \\
\hline$A$ & Biofilm surface area & 240 & $\mathrm{~m}^{2}$ & chosen \\
\hline$L_{f}$ & Biofilm thickness & 500 & $\mu \mathrm{m}$ & chosen \\
\hline$K_{L} a$ & $\begin{array}{l}\text { Total mass transfer coefficient for } \\
\text { oxygen }\end{array}$ & 0.312 & $\mathrm{~m} / \mathrm{d}$ & chosen \\
\hline$F_{\text {in }}$ & Influent flow rate & 0.24 & $\mathrm{~m}^{3} / \mathrm{d}$ & chosen \\
\hline$G_{\text {in }}$ & Airflow rate & 1.3 & $\mathrm{~m}^{3} / \mathrm{d}$ & chosen \\
\hline$H R T$ & Hydraulic retention time & 4 & day & calculated \\
\hline$S_{N H 4, \text { in }}$ & Influent $\mathrm{NH}_{4}^{+}$concentration & 1000 & $\mathrm{~g}-\mathrm{N} / \mathrm{m}^{3}$ & chosen \\
\hline$T$ & Temperature & 30 & ${ }^{\circ} \mathrm{C}$ & chosen \\
\hline
\end{tabular}




\section{References:}

1. Kinh, C. T.; Riya, S.; Hosomi, M.; Terada, A., Identification of hotspots for NO and N2O production and consumption in counter- and co-diffusion biofilms for simultaneous nitrification and denitrification. Bioresour. Technol. 2017, 245, 318-324.

2. Hiatt, W. C.; Grady, C. P. L., An Updated Process Model for Carbon Oxidation, Nitrification, and Denitrification. Water Environ. Res. 2008, 80, (11), 2145-2156.

3. Strous, M.; Heijnen, J. J.; Kuenen, J. G.; Jetten, M. S. M., The sequencing batch reactor as a powerful tool for the study of slowly growing anaerobic ammonium-oxidizing microorganisms. Appl. Microbiol. Biotechnol. 1998, 50, (5), 589-596.

4. Ni, B. J.; Ye, L.; Law, Y. Y.; Byers, C.; Yuan, Z. G., Mathematical Modeling of Nitrous Oxide (N2O) Emissions from Full-Scale Wastewater Treatment Plants. Environ. Sci. Technol. 2013, 47, (14), 7795-7803.

5. Ni, B. J.; Ruscalleda, M.; Pellicer-Nacher, C.; Smets, B. F., Modeling Nitrous Oxide Production during Biological Nitrogen Removal via Nitrification and Denitrification: Extensions to the General ASM Models. Environ. Sci. Technol. 2011, 45, (18), 7768-7776.

6. Wiesmann, U., Biological nitrogen removal from wastewater. In Biotechnics/Wastewater, Springer Berlin Heidelberg: 1994; Vol. 51, pp 113-154.

7. Koch, G.; Egli, K.; Van der Meer, J. R.; Siegrist, H., Mathematical modeling of autotrophic denitrification in a nitrifying biofilm of a rotating biological contactor. Water Sci. Technol. 2000, 41, (4-5), 191-198.

8. Hao, X.; Heijnen, J. J.; van Loosdrecht, M. C. M., Sensitivity analysis of a biofilm model describing a one-stage completely autotrophic nitrogen removal (CANON) process. Biotechnol. Bioeng. 2002, 77, (3), 266-277.

9. Haynes, W. M., CRC handbook of chemistry and physics. CRC press: 2014.

10. Sabba, F.; Picioreanu, C.; Pérez, J.; Nerenberg, R., Hydroxylamine Diffusion Can Enhance N2O Emissions in Nitrifying Biofilms: A Modeling Study. Environ. Sci. Technol. 2015, 49, (3), 1486-1494.

11. Zacharia, I. G.; Deen, W. M., Diffusivity and solubility of nitric oxide in water and saline. Ann. Biomed. Eng. 2005, 33, (2), 214-222.

12. Horn, H.; Morgenroth, E., Transport of oxygen, sodium chloride, and sodium nitrate in biofilms. Chem. Eng. Sci. 2006, 61, (5), 1347-1356. 